\title{
Respuesta a antidepresivos serotoninérgicos y noradrenérgicos: estudio cruzado con fluoxetina y desipramina en pacientes con un primer episodio depresivo mayor
}

José Alfonso Ontiveros-Sánchez de la Barquera

Departamento de Psiquiatría, Hospital Universitario, Universidad Autónoma de Nuevo León; Instituto de Información e Investigación en Salud Mental A.C., Monterrey, N.L., México

\begin{abstract}
Resumen
Antecedentes: Las tasas de respuesta a diferentes clases de antidepresivos ayudan a elaborar guías para la prescripción racional en farmacoterapia. Sin embargo, existen pocos estudios comparativos con antidepresivos inhibidores selectivos de la recaptación de serotonina o noradrenalina en la misma muestra de pacientes con depresión mayor. Método: Pacientes ambulatorios con un primer episodio depresivo mayor (DSM-III-R) que completaron 6 semanas de tratamiento en dos estudios a doble ciego aleatorizados con fluoxetina y desipramina que recibieron por 6 semanas de manera cruzada el otro fármaco antidepresivo. Como respuesta se consideró si el puntaje final de la escala Hamilton de depresión disminuyó un $50 \%$ o más desde la determinación basal. Resultados: No encontramos diferencias por tratamiento o secuencia. Diez de los 18 pacientes (55.5\%) respondieron a fluoxetina y desipramina, tres(16.6\%) fueron resistentes para fluoxetina y tres (16.6\%) para Desipramina, y dos (11.1\%) para ambos fármacos. Discusión: Nuestro estudio sugiere que, en pacientes ambulatorios con un primer episodio depresivo, la fluoxetina y la desipramina son igualmente eficaces. En los pacientes que no respondieron a uno de los fármacos estudiados, el otro tratamiento fue eficaz; esta maniobra de tratamiento debe ser considerada en estos pacientes.
\end{abstract}

PALABRAS CLAVE: Fluoxetina. Desipramina. Depresión mayor. Primer episodio. Estudio cruzado.

\begin{abstract}
Background: Response rate data from studies with different kinds of antidepressant drugs help in the development of guidelines for the rational prescription of pharmacotherapy. However, there are still few comparative studies with selective reuptake inhibition on serotonin or norepinephrine in the same sample of major depression patients. Methods: First episode major depression (DSM-III-R) outpatients who completed 6 weeks in two double-blind randomized trials with fluoxetine and desipramine were crossed over to treatment with the other drug under open conditions for 6 weeks. Response was considered if patient's final Hamilton depression scale score decreased $50 \%$ or more from baseline. Results: No significant differences were found by drug treatment or sequence of treatment. Ten of the 18 patients (55.5\%) were responders to both fluoxetine and desipramine, $3(16.6 \%)$ were resistant to fluoxetine, $3(16.6 \%)$ to desipramine and $2(11.1 \%)$ to both drugs. Discussion: These data suggest that among first major depressive episode outpatients fluoxetine and desipramine are equally effective. In patients who have been non-responders to one of the studied drugs, the other one is strikingly effective; this kind of treatment maneuver should be considered in such patients.
\end{abstract}

KEY WORDS: Fluoxetine. Desipramine. Major depression. First episode. Crossover study.

\author{
Correspondencia: \\ José Alfonso Ontiveros-Sánchez de la Barquera \\ Dr. Enrique Peña, 122, 2. ${ }^{\circ}$ piso \\ Col. Doctores \\ C.P. 64710 , Monterrey, N.L., México \\ E-mail: ontiverosalf@gmail.com
}

Fecha de recepción: 13-07-2016

Fecha de aceptación: 28-11-2016

DOI:10.24875/GMM.17002671
Gac Med Mex. 2017;153:688-694

Contents available at PubMed www.gacetamedicademexico.com 


\section{Introducción}

Diez años después de la aparición de los primeros antidepresivos (inhibidores de la monoaminooxidasa y tricíclicos) y con los avances alcanzados, Joseph J. Schildkraut (1934-2006) publicó en 1965 lo que hoy conocemos como la hipótesis catecolaminérgica de la depresión ${ }^{1}$. Desde su publicación, esta teoría que asocia la depresión a bajos niveles de neurotransmisores fue aceptada para explicar no solo la neurobioquímica de la depresión, sino también el efecto de los fármacos antidepresivos. Esta teoría revolucionó nuestra comprensión sobre la función de los neurotransmisores en los trastornos afectivos cimentando las bases para su investigación básica y clínica. En breve, esta hipótesis postula que, en la depresión, la función de las monoaminas dopamina, noradrenalina e indolamina serotonina se encuentra disminuida ${ }^{2}$. En sustento a lo anterior, diversos estudios han mostrado cambios en las concentraciones plasmáticas, urinarias y en líquido cefalorraquídeo de estos neurotransmisores y sus metabolitos, cambios en la densidad de neurorreceptores en plaquetas y neuronas, curvas aplanadas en retos neuroendocrinos y recaídas tempranas con el bloqueo de enzimas de restricción para la síntesis de neurotransmisores en pacientes que habían remitido de la depresión con un tratamiento antidepresivo ${ }^{3}$. Se ha postulado también la existencia de depresiones predominantemente deficitarias noradrenérgicas, serotoninérgicas 0 dopaminérgicas. Los pacientes con estos subtipos de depresiones hipotéticamente responderían mejor a fármacos antidepresivos con efectos noradrenérgicos, serotoninérgicos y dopaminérgicos, según el caso. Sin embargo, estudios clínicos sobre el efecto de antidepresivos con diferentes mecanismos de acción muestran resultados contradictorios y no existen parámetros biológicos o clínicos claros que puedan predecir los resultados de diferentes tratamientos antidepresivos ${ }^{4}$. Cabe señalar que, a la luz de los conocimientos actuales, la teoría catecolaminérgica parece simplista y ha sido objeto de numerosas críticas y revisiones. Hoy en día, de hecho, es muy aventurado pensar que el déficit de un solo neurotransmisor estaría presente en la mayoría de los pacientes deprimidos. Sin embargo, sigue siendo importante conocer si existen grupos de pacientes que puedan responder mejor a fármacos con diferentes mecanismos de acción. Sin embargo, los estudios cruzados en los que el mismo paciente es expuesto a dos fármacos para diferenciar las respuestas a antidepresivos con diferentes mecanismos de acción son escasos en la literatura. Empero, esta aproximación ha sido empleada en grupos de pacientes con trastorno obsesivo compulsivo ${ }^{5,6}$ y trastorno dismórfico corporal ${ }^{7}$, encontrando que responden mejor a los fármacos antidepresivos con efecto serotoninérgico, pero no noradrenérgico.

El presente estudio cruzado fue diseñado con la hipótesis de que existen subgrupos de pacientes con trastorno depresivo mayor que responden mejor a antidepresivos con efecto serotoninérgico o noradrenérgico. Para lo mismo sometimos en forma cruzada a dos grupos de pacientes a los antidepresivos fluoxetina, un inhibidor selectivo de la recaptación de la serotonina (ISRS), y desipramina, un tricíclico inhibidor potente de la recaptura de noradrenalina (ISRN). Seleccionamos pacientes que cursaban con un primer episodio depresivo mayor sin antecedentes de tratamientos antidepresivos previos. Un grupo de pacientes fue asignado primero a fluoxetina y luego a Desipramina, y otro recibió primero desipramina y después fluoxetina. Cada fármaco fue proporcionado por 6 semanas.

\section{Método}

Se incluyeron pacientes de entre 18 y 65 años de edad, que reunían los criterios del DSM-III-R ${ }^{8}$ para un primer episodio depresivo mayor, sin experiencia previa con tratamientos antidepresivos ni tratamiento con fármacos psicotrópicos concomitantes. Los pacientes debían tener una enfermedad de gravedad moderada (impresión clínica global de gravedad mayor o igual a 4 puntos) y un puntaje de 18 o más en la escala de Hamilton para la depresión. Se excluyeron pacientes con otros diagnósticos psiquiátricos, incluyendo cualquier trastorno psicótico, esquizofrenia, trastorno bipolar, trastornos de ansiedad, abuso o dependencia al alcohol o drogas (DSM-III-R), enfermedades médicas inestables o relevantes, pacientes con embarazo o lactancia (todas las mujeres en edad fértil debían contar con un método anticonceptivo de doble barrera), y pacientes en psicoterapia o con riesgo suicida. Los pacientes debían tener 2 semanas o más sin tratamientos psicotrópicos. Los participantes en el estudio fueron incluidos a doble ciego en dos grupos, uno en el que recibieron fluoxetina y otro desipramina, y al terminar fueron invitados para participar en el estudio cruzado. Todos los pacientes firmaron una carta de consentimiento antes de ser incluidos en el estudio. En una primera fase del estudio, los pacientes recibieron por al menos una semana (máximo dos) tratamiento con placebo 
a simple ciego. Al final de esta fase, aquellos pacientes que continuaron con 18 o más puntos en la escala de Hamilton para la depresión en los primeros 17 ítems, y que no mejoraron un $20 \%$ o más, recibieron tratamiento activo. El grupo de pacientes que tomó fluoxetina por 6 semanas recibió 6 semanas de desipramina, y el otro grupo que tomó primero desipramina recibió fluoxetina por igual lapso de tiempo. La gravedad de la depresión y su evolución clínica fueron estudiadas con las escalas HAMD de 21 puntos, impresión clínica global de gravedad (ICG-S) e impresión clínica global de mejoría (ICG-M) de 8 puntos. La escala HAMD se completó en la visita de selección antes de iniciar el tratamiento, después de la fase de placebo a simple ciego y al final de cada etapa del tratamiento cruzado de 6 semanas. Las escalas ICG-S e ICG-M fueron completadas en la visita de inicio y en los días 14, 28 y 42 de cada curso de tratamiento. Los pacientes con una reducción igual o mayor del $50 \%$ del puntaje basal en la escala HAMD y una calificación en la ICG-M equivalente a «mejoría» o «mucha mejoría» fueron considerados pacientes respondedores. Se consideraron para las evaluaciones finales solo aquellos pacientes que completaron en el estudio 84 días de tratamiento continuo (42 días con fluoxetina y 42 días con desipramina). La fluoxetina se proporcionó a una dosis fija de $20 \mathrm{mg}$ al día por la mañana y la desipramina fue administrada por las noches de acuerdo con el siguiente esquema: $25 \mathrm{mg}$ los primeros 3 días, $50 \mathrm{mg}$ por 4 días más, y $100 \mathrm{mg}$ por 1 semana seguido de aumentos opcionales de $50 \mathrm{mg}$ cada semana hasta alcanzar una dosis máxima de $250 \mathrm{mg}$ al día, buscando el máximo de mejoría y tolerabilidad. El análisis estadístico de los datos categóricos se realizó con la prueba de ji al cuadrado para datos continuos, y se empleó también la prueba de comparación de dos proporciones. Para comparar los datos de los pacientes en los dos tratamientos estudiados se emplearon las pruebas de Mann-Whitney ot de Student. Se utilizó la prueba de Kruskal-Wallis para comparar los valores de las escalas HAMD e ICG-S basales con los valores obtenidos durante el curso de los tratamientos. Las proporciones son reportadas con sus desviaciones estándar.

\section{Resultados}

Ingresaron al estudio 23 pacientes que habían completado 6 semanas de tratamiento (12 pacientes con fluoxetina y 11 pacientes con desipramina). Estos 23 pacientes tuvieron una edad de $36.6 \pm 12.89$ años, $12(66.6 \%)$ eran mujeres, $14(77.7 \%)$ casados/as y 3 pacientes $(16.6 \%)$ tenían estudios profesionales. De los 23 pacientes, 18 completaron las 12 semanas de tratamiento cruzado, 9 recibieron la secuencia desipramina-fluoxetina y 9 la secuencia fluoxetina-desipramina. Ambos grupos no difirieron en edad, distribución por sexo, signos vitales (excepto la presión diastólica), peso, gravedad de la depresión y duración del episodio depresivo (Tabla 1).

Los 18 pacientes que completaron el estudio cruzado y los cinco que lo terminaron prematuramente no difirieron en cuanto a variables demográficas y clínicas. Sin embargo, los pacientes que recibieron primero fluoxetina tuvieron una depresión más grave $(26.2+3.9)$ que aquellos que recibieron desipramina $(21.5+3.7)$ (Mann-Whitney's $z=2.66, p=0.01$ ) en la escala HAMD 21 , sin que hubiera diferencias entre ambos grupos antes de la fase de placebo a simple ciego. Luego de la desipramina, los pacientes que recibieron fluoxetina tuvieron puntajes menores en la escala ICG-S $(p<0.05)$.

Ambos grupos de pacientes estudiados mostraron a lo largo del estudio una reducción estadísticamente significativa en los puntajes de las escalas HAMD e ICG-S y mejoría (Tabla 2 y Figs. 1 y 2). Los pacientes considerados como respondedores de acuerdo con los criterios del estudio fueron 13 para fluoxetina y 13 para desipramina (secuencia fluoxetina [7] y desipramina [9]; secuencia desipramina [4] fluoxetina [6]). De los 18 pacientes estudiados, $10(55.5 \%)$ respondieron a los dos antidepresivos estudiados, 2 (11.1\%) respondieron solamente al ISRS fluoxetina y 2 al ISRN desipramina $(11.1 \%)$. Tres pacientes $(16.6 \%)$ fueron no respondedores a la fluoxetina y $3(16.6 \%)$ a la desipramina, y 2 pacientes (11.1\%) no respondieron a ambos antidepresivos. La tabla 3 muestra los puntajes finales de la escala ICG-S en el grupo de pacientes respondedores y no respondedores al final de las semanas 6 y 12 para ambas secuencias estudiadas.

Los 18 pacientes que completaron el estudio recibieron la dosis siguiente de tratamiento de desipramina: secuencia fluoxetina-desipramina $188.8 \pm 33.3 \mathrm{mg} / \mathrm{día}$, secuencia desipramina-fluoxetina $205.5 \pm 48.1 \mathrm{mg} / \mathrm{día}$; el rango de dosis fue de 125 a $250 \mathrm{mg} / \mathrm{día}$ ( $\mathrm{t}=82$; $d f=14 ; p>0.17$, no significativo). Todos los pacientes fueron tratados con $20 \mathrm{mg}$ al día de fluoxetina. De los 23 pacientes que iniciaron el estudio, las razones para la terminación prematura fueron: eventos adversos (dos pacientes con fluoxetina y uno con desipramina), falta de eficacia y efectos adversos (un paciente con fluoxetina) y pérdida de seguimiento (un paciente 
Tabla 1. Diferencias demográficas y clínicas de los pacientes tratados por secuencia de tratamiento, antes del tratamiento

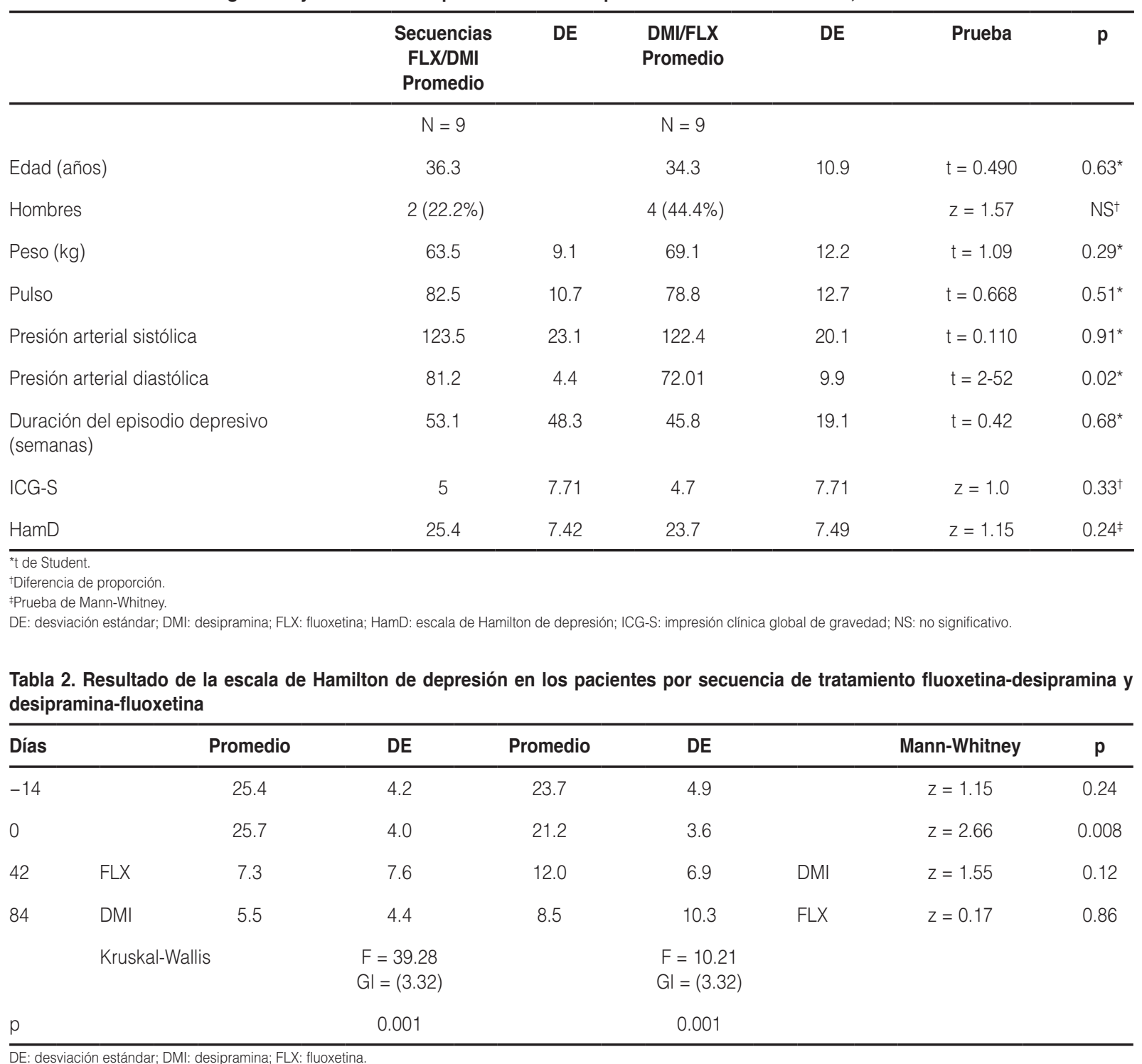

DE: desviación estándar; DMI: desipramina; FLX: fluoxetina.

con desipramina). Con la desipramina observamos un cambio estadísticamente significativo en la frecuencia cardiaca $(95.3 \pm 5.4)$ en comparación con la fluoxetina $(76.1 \pm 9.1 ; t=5.08 ; d f=13 ; p=0.001)$. La tabla 4 muestra los eventos adversos observados durante el estudio y el tiempo en que se alcanzaron diferencias estadísticamente significativas. Para cada evento adverso reportado con desipramina hubo 1.73 eventos adversos con fluoxetina (prueba de proporción $p<0.05$ ). Los eventos adversos más frecuentes con la fluoxetina fueron cefalea (a la semana 4), insomnio y bochornos (a la semana 8), y nerviosismo (a la semana 10). La xerostomía fue el único evento adverso estadísticamente más frecuente con la desipramina (a las semanas 10 y 12). Eventos adversos de intensidad grave fueron reportados por seis pacientes, tres con fluoxetina y tres con desipramina.

\section{Discusión}

En la depresión mayor, los escasos estudios cruzados publicados han explorado la existencia de subgrupos de pacientes que podrían responden mejor a fármacos con efecto serotoninérgico o noradrenérgico. Algunos de estos estudios han incluido variables neurobioquímicas y clínicas que podrían diferenciar entre grupos de pacientes. Así, en un estudio con 65 pacientes hospitalizados con depresión "endógena» se detectaron bajos valores de dos metabolitos de la 


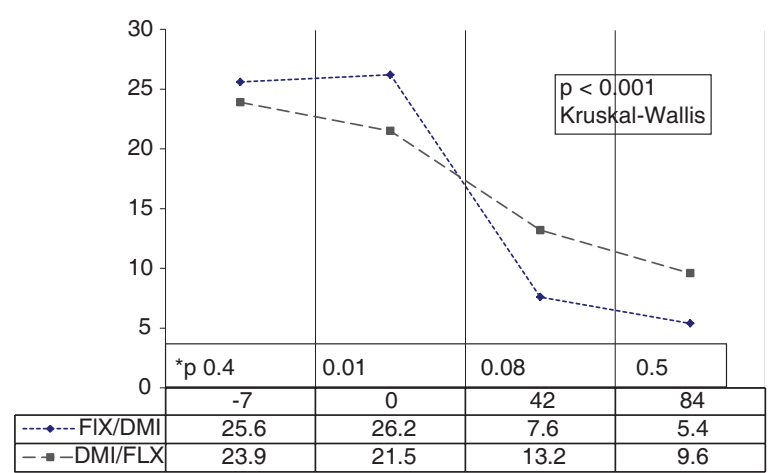

Figura 1. Comparación de los puntajes de la escala de Hamilton para la depresión en los pacientes tratados con las secuencias fluoxetina-desipramina (FLX/DMI) y desipramina-fluoxetina (DMI/FLX).

*Prueba de Mann-Whitney.

serotonina (5-HIAA y HVA) en el líquido cefalorraquídeo en los pacientes que respondieron mejor al ISRS zimelidina, en tanto que los pacientes con valores altos de HMPG (un metabolito de la noradrenalina) en el líquido cefalorraquídeo respondieron mejor a la desipramina ${ }^{9-14}$. Se ha sugerido que existen subgrupos de pacientes depresivos con bajos valores centrales de serotonina y pacientes con una tendencia a valores más altos de HVA que responden mejor al ISRN desipramina $^{9,14,15}$, aunque estas observaciones no han sido confirmadas en todos los estudios ${ }^{16}$.

Varios estudios cruzados han proporcionado el segundo tratamiento solo a grupos de pacientes resistentes al primer antidepresivo administrado, o han incluido poblaciones mixtas de pacientes depresivos y con depresión bipolar ${ }^{17}$. Así, el primer estudio cruzado a doble ciego reportado en la literatura efectuado con

Tabla 3. Respuesta al tratamiento con fluoxetina y desipramina

\begin{tabular}{|c|c|c|c|c|}
\hline \multirow[t]{2}{*}{ ICG-M } & \multicolumn{2}{|c|}{$\begin{array}{l}\text { Fluoxetina } \\
\text { Desipramina }\end{array}$} & \multicolumn{2}{|c|}{$\begin{array}{l}\text { Desipramina } \\
\text { Fluoxetina }\end{array}$} \\
\hline & $\mathrm{N}(\%)$ & $\mathrm{N}(\%)$ & $\mathrm{N}(\%)$ & $\mathrm{N}(\%)$ \\
\hline Mucho muy mejorado & $7(78)$ & $8(89)$ & $3(33)$ & $6(67)$ \\
\hline Muy mejorado & $1(11)$ & $1(11)$ & $2(22)$ & $1(11)$ \\
\hline Mejoría leve & $1(11)$ & 0 & $3(33)$ & 0 \\
\hline Sin cambio & 0 & 0 & 0 & $2(22)$ \\
\hline Levemente peor & 0 & 0 & $1(11)$ & 0 \\
\hline $\begin{array}{l}\text { Respondedores } \\
\text { HamD }<50 \% \text { desde basal }\end{array}$ & $7(78)$ & $9(100)$ & $4(44.5)$ & $6(67)$ \\
\hline $\begin{array}{l}\text { No respondedores } \\
\text { HamD } \geq 50 \% \text { desde basal }\end{array}$ & $2(22)$ & $0(0)$ & $5(55.5)$ & $3(33)$ \\
\hline
\end{tabular}


más importante cuando un fármaco ha fallado en mejorar al paciente y se debe proporcionar uno alternativo, cuando los efectos adversos fuerzan el cambio del tratamiento o cuando se considera la combinación de dos antidepresivos. Aunque no existe un consenso, en diversos estudios comparativos con antidepresivos con efectos predominantemente serotoninérgicos 0 noradrenérgicos se ha observado que los pacientes con mayor retardo psicomotor no responden bien a fármacos serotoninérgicos, pero sí a fármacos noradrenérgicos ${ }^{18,19}$. Entre estos casos se encuentran pacientes con depresiones melancólicas ${ }^{20,21}$. Se ha reportado que buenas respuestas al fármaco noradrenérgico maprotilina se correlacionan con un menor número de episodios depresivos previos y duración en años desde el primer episodio, en tanto que con zimelidina los mejores resultados se observaron cuando los pacientes tenían más episodios depresivos previos ${ }^{15}$; sin embargo, estos datos no han sido corroborados en otros estudios ${ }^{22}$.

La tasa de respuesta antidepresiva general a cualquier fármaco es cercana al $75 \%{ }^{23}$. Nuestro estudio cruzado apoya la observación de que podríamos clasificar a los pacientes depresivos por sus respuestas a los medicamentos serotoninérgicos y noradrenérgicos en tres subgrupos: 1) un grupo mayor de casos (55\% en nuestro estudio) que responden a ambos tipos de antidepresivos; 2) un grupo de pacientes (22\% en nuestro estudio) que responden a uno solo de ellos (ISRS 11\%, ISRN 11\%), y 3 ) un grupo de pacientes resistentes a ambos fármacos $(11 \%$ en nuestro estudio). Al sumar los pacientes que respondieron a ambos medicamentos (55\%) y aquellos que solo respondieron a uno $(22 \%)$, la eficacia antidepresiva para la fluoxetina y la desipramina fue del $77.7 \%$. Cabe señalar que esta tasa es superior a la reportada por Bowden, et al. ${ }^{24}$ en un estudio doble ciego de 6 semanas en el que respondieron a la fluoxetina el $64 \%$ y a la desipramina el $68 \%$. Lingjaerde et al. ${ }^{12}$ y Äberg-Wistedt ${ }^{10}$ encontraron una tendencia bimodal de respuesta (todo o nada) al ISRS zimelidina y a la desipramina en estudios cruzados a doble ciego; situación que no encontramos en nuestro estudio y que puede ser explicada por tratarse de una población diferente de pacientes. La respuesta de pacientes resistentes a fármacos ISRS e ISRN merece ser comentada en el contexto del presente trabajo. Los reportes en la literatura muestran tasas de respuesta del 43 al $75 \%$ cuando los pacientes resistentes a un ISRS son cambiados a un ISRN, y viceversa ${ }^{23}$. Emrich, et al. ${ }^{22}$, en 71 pacientes resistentes a antidepresivos tricíclicos, compararon el ISRS fluvoxamina y el ISRN oxaprotilina. Los pacientes no respondedores fueron cruzados al otro tratamiento y se obtuvieron respuestas del 33\% (21 de 64 pacientes) con oxaprotilina, pero solo del $4 \%$ (2 de 56 pacientes) con fluvoxamina. En contraste, un estudio comparativo con maprotilina ( $N=35$ ) y zimelidina $(N=40)$, en el que los pacientes que no mejoraron fueron cambiados al otro tratamiento, encontró que siete pacientes en cada grupo no respondieron a 4 semanas de tratamiento (20\% para maprotilina y $17.5 \%$ para zimelidina), y de estos, mejoraron seis pacientes en cada grupo (17\% con maprotilina y $15 \%$ con zimelidina $)^{12}$. White, et al..$^{19}$, en 12 pacientes resistentes a la desipramina, observaron que al ser cambiados a doble ciego al ISRS fluvoxamina mejoraron el $75 \%$, y Delgado, et al. ${ }^{25}$, en un estudio abierto con siete pacientes que no respondieron a la desipramina, reportaron respuesta en el $71 \%$ al ser tratados con fluvoxamina. Pacientes resistentes a los antidepresivos tricíclicos responden en un $60 \%$ cuando son cambiados a un ISRS, y el $65 \%$ de los pacientes resistentes a un ISRS mejoran con tricíclicos ISRN como nortriptilina, desipramina u oxtremolina ${ }^{21,26-28}$. En nuestro estudio, el $75 \%$ de los pacientes resistentes a la desipramina mejoraron con fluoxetina, y el $75 \%$ de los resistentes a la fluoxetina mejoraron con desipramina. Nuestros resultados coinciden con los estudios en pacientes resistentes.

Nuestros hallazgos en este estudio cruzado de 12 semanas con fluoxetina y desipramina deben ser evaluados a la luz de las limitaciones de tratarse de un estudio abierto, con una muestra pequeña, como suele suceder en los estudios cruzados, en el que hubo un $21.7 \%$ de terminaciones prematuras. Además, no evaluamos las concentraciones plasmáticas de desipramina, si bien los pacientes recibieron la dosis máxima tolerada de 205.5 mg por día en promedio (rango: 125 a $250 \mathrm{mg} / \mathrm{día}$ ). Desconocemos si una dosis mayor de $20 \mathrm{mg}$ de fluoxetina podría haber mejorado a un mayor número de pacientes. Es probable que en los pacientes que recibieron primero fluoxetina y luego desipramina las concentraciones plasmáticas de la desipramina hayan sido mayores por al menos 3 semanas $^{29,30}$, por inhibición de la citocromo P450 subunidad 2D6 de la fluoxetina y su metabolito la norfluoxetina. En concordancia, eventos adversos como bochornos e insomnio se presentaron más cuando los pacientes fueron cambiados de fluoxetina a desipramina. Empero, el cambio rápido de antidepresivo es una maniobra recomendada y bien tolerada ${ }^{31}$. Finalmente, no puede descartarse, 
como sucede en los estudios cruzados, un efecto de «acarreo" del efecto del primer tratamiento otorgado, evidenciado por una disminución progresiva estadísticamente significativa en las escalas de Hamilton para la depresión e ICG-S con ambas secuencias de tratamiento.

El presente estudio sobre los efectos antidepresivos diferenciados de los ISRS e ISRN da luz sobre las respuestas a estos agentes farmacológicos y apoya que existen cuadros depresivos que responden mejor a fármacos noradrenérgicos y serotoninérgicos, y pueden orientar al clínico en la selección del tratamiento antidepresivo en pacientes resistentes a los ISRN e ISRS. Se requieren más estudios cruzados con diferentes antidepresivos con efectos serotoninérgicos y noradrenérgicos para confirmar nuestros hallazgos.

\section{Financiamiento}

Apoyado en parte por el Sistema Nacional de Investigadores 11503.

\section{Bibliografía}

1. Green Al, Schatzberg AF. Obituary. Joseph J. Schildkraut, 1934-2006 Neuropsychopharmacology. 2007;32:1855-6.

2. Schildkraut, JJ. The catecholamine hypothesis of affective disorders: a review of supporting evidence. Am J Psychiatry. 1965;122:509-22.

3. Goldstein DJ, Potter WZ, Ciraulo DA, et al. Biological theories of depression and implications for current and new treatments. En: Ciraulo DA Shader RI, editores. Pharmacotherapy of depression. $2^{\text {nd }}$ ed. New York: Human Press; 2011. p. 1-32.

4. Humble M. Noradrenaline and serotonin reuptake inhibition as clinica principle: a review of antidepressant efficacy. Acta Psychiatr Scand. 2000;101(Suppl 402):28-36

5. Riddle MA, Scahill L, King RA, et al. Double-blind, crossover trial of fluoxetine and placebo in children and adolescents with obsessive-compulsive disorder. J Am Acad Child Adolsc Psychiatr. 1992;31:1062-9.

6. Goodman WK, Price LH, Delgado PL, et al. Specificity of serotonin reuptake inhibitors in the treatment of obsessive-compulsive disorder. Comparison of fluvoxamine and desipramine. Arch Gen Psychiatry. 1990;47:577-85.

7. Hollander E, Allen A, Kwon J, et al. Clomipramine vs desipramine crossover trial in body dysmorphic disorder. Selective efficacy of a serotonin reuptake inhibitor in imagined ugliness. Arch Gen Psychiatry. 1999;56:1033-9.

8. American Psychiatric Association. Diagnostic and statistical manual of mental disorders. $3^{\text {rd }}$ edition-revisited. Washington, DC: American Psychiatric Press; 1987.
9. Äberg-Wisted A, Jostell KG, Ross SB, et al. Effects of zimelidine and desipramine on serotinin and noradrenaline uptake mechanism in relation to plasma concentrations and to therapeutic effects during treatment of depression. Psychopharmacology. 1981;74:297-305.

10. Äberg-Wistedt A. A double blind study of zimelidine, a serotonin uptake inhibitor, and desipramine, a noradrenaline uptake inhibitor, in endogenous depression. I. Clinical finding. Acta Psychiatr Scand. 1982;66:50-65.

11. Äberg-Wistedt A. Comparison between zimelidine and desipramine in endogenous depression. A cross-over study. Acta Psychiatr Scand. 1982;66:129-38.

12. Lingjaerde $\mathrm{O}$, Bratfos $\mathrm{O}$, Bratlid $\mathrm{T}$, et al. A double-blind comparison of zimelidine and desipramine in endogenous depression. Acta Psychiatr Scand. 1983:68:22-33

13. Nystgröm $\mathrm{C}$, Hällström T. Comparison between a serotonin and a noradrenaline reuptake blocker in the treatment of depressed outpatients. A cross-over study. Acta Psychiatr Scand. 1987;75:377-82.

14. Äberg A. Controlled crossover study of a $5 \mathrm{HT}$ uptake inhibiting and an NA uptake inhibiting antidepressant. Acta Psychiatr Scand. 1981;63(Suppl 190):224-55

15. van Praag HM. New evidence of serotonin-deficient depressions. Neuropsychobiology. 1977;3:56-63

16. Burns RA, Lock T, Edwards DR, et al. Predictors of response to amine-specific antidepressants. J Affect Disord. 1995;35:97-106.

17. Potter WZ, Calil HM, Extein I, et al. Specific norepinephrine and serotonin uptake inhibitors in man: a crossover study with pharmacokinetic, biochemical, neuroendocrine and behavioral parameters. Acta Psychiatr Scand Suppl. 1981;290:152-65.

18. Nyström C, Hällström T. Comparison between a serotonin and a noradrenaline reuptake blocker in the treatment of depressed outpatients. A cross-over study. Acta Psychiatr Scand. 1987;75:377-82

19. White K, Wykoff W, Tyness LL, et al. Fluovoxamine in the treatment of tricyclic-resistant depression. J Psychatr Neurosci. 1990;15:156-8.

20. Szegedi A, Wetzel H, Angersbach $D$, et al. A double-blind study comparing paroxetine and maprotiline in depressed outpatients. Pharmacopsychiatry. 1997;30:97-105.

21. Nolen WA, van de Putte JJ, Dijken WA, et al. Treatment strategy in depression. I. Non-tricyclic and selective reuptake inhibitors in resistant depression: a double-blind partial crossover study on the effects of oxaprotiline and fluvoxamine. Acta Psychiatr Scand. 1988;78:668-75.

22. Emrich $\mathrm{H}$, Berger $\mathrm{M}$, Riemann $\mathrm{D}$, et al. Serotonin reuptake inhibition versus norepinephrine reuptake inhibition: a double-blind differential therapeutic study with fluvoxamine and oxaprotiline in endogenous and neurotic depressives. Pharmacopsychiatrica. 1987;20:60-3.

23. Thase ME, Rush AJ. Treatment-resistant depression. En: Bloom FE, Kupfer DJ, editors. Psychopharmacology, the fourth generation of progress. New York: Raven Press; 1995. p. 1081-97.

24. Bowden CL, Schatzberg AF, Rosenbaum A, et al. Fluoxetine and desipramine in major depressive disorder. J Clin Psychopharmacol. 1993; 135:305-11.

25. Delgado PL, Price LH, Charney DS, et al. Efficacy of fluovoxamine in treatment-refractory depression. J Affect Disord. 1988;15:55-60.

26. Souery D, Serretti A, Calati R, et al. Citalopram versus desipramine in treatment resistant depression: effect of continuation or switching strategies. A randomized open study. World J Biol Psych. 2011;12:364-73.

27. Papakostas GI, Fava M. Pharmacotherapy for depression and treatment-resistant depression. USA: World Scientific Publishing Co. Pte. Ltd; 2010.

28. Nierenberg AA, Papakostas GI, Petersen T, et al. Nortriptyline for treatment-resistant depression. J Clin Psychiatry. 2003;64:35-9.

29. Suckow RF, Roose SP, Cooper TB. Effect of fluoxetine on plasma desipramine and 2-hidroxydesipramine. Biol Psychiatry. 1992;31(2 Suppl): 200-4.

30. Preskorn SH, Alderman J, Chung M, et al. Pharmacokinetics of desipramine coadministered with sertraline or fluoxetine. J Clin Psychopharmacol. 1994:14(2 Suppl): $90-8$

31. Van Ameringen M, Mancini C. Adverse effects of switching from fluoxetine to desipramine (letter). Can J Psychiatry. 1992;37(4 Suppl):278. 